\title{
Influence of Injection/Suction on Mixed Convection Flow Across a Vertical Cone Saturated Porous Medium with Double Dispersion and Chemical Reaction Effects
}

\author{
Om Prakash Meena \\ Department of Mathematics, Shaheed Bhagat Singh College, University of Delhi, New Delhi 110017, India
}

Corresponding Author Email: omi.omprakash95@gmail.com

https://doi.org/10.18280/ti-ijes.652-442

Received: 22 March 2021

Accepted: 27 May 2021

\author{
Keywords: \\ mixed convection, vertical cone, \\ injection/suction effect, double dispersion \\ effect, chemical reaction effect, bivariate \\ Chebyshev spectral collocation quasi- \\ linearization method (BSCQLM)
}

\begin{abstract}
We examined how injection/suction impacts the flow characteristics for mixed convection across a vertical cone saturated porous medium in the presence of double dispersion and chemical reaction effects. We perform suitable transformations to convert the nonlinear system of partial differential expressions into a system of non-dimensional form and received dimensionless equations solved numerically by the bivariate Chebyshev spectral collocation quasi-linearization method. We explain the outcomes of the flow characteristics over various variables through diagrams and numerical benchmarks. We also establish precision verification of the chosen numerical technique through a comparison with prior published computations and found to be in great assent. The residual analysis section also illustrated which unblocks convergence of the present results.
\end{abstract}

\section{INTRODUCTION}

Flow across cone-shaped frames ordinarily encountered which has brought significant attention in many engineering applications, the physical phenomenon over a vertical cone takes place in heat exchangers, geothermal reservoirs, nuclear reactor cooling systems and style of canisters for nuclear waste disposal, etc. Mixed convection flow through saturated porous media is another essential field that attracted in the eyes of the researcher's many researchers who analyzed it on various geometries. Magnetohydrodynamic study over a vertical plated was proposed by Aldoss et al. [1]. Khanafer and Chamkha [2] introduce flow in a lid-driven filled with fluid. Flow over a vertical wedge offered by Kumari et al. [3]. Aydın and Kaya [4] introduced MHD flow over a permeable vertical plate. A fully developed flow between inclined parallel plates presented by Cimpean et al. [5]. Mukhopadhyay [6] offered flow along with a stretching cylinder and we can view more appropriate work as [7-11].

Mass transfer through a wall slot into the boundary layer is enthusiastic because of several prospective applications including thermal protection, energizing the inner section of the boundary layer region in adverse pressure gradient and skin friction reduction in control surfaces. Mass transfer into a slot completely impacts the progress of the boundary layer along the surface and can inhibit or at least limit the separation of the viscous region. Some analysts offered it on various bodies. Flow in a non-Darcian fluid-saturated porous enclosure under multiple suction effects was offered by Kumar and Murthy [12]. Daba and Devaraj [13] analyzed over a vertical stretching sheet of unsteady double-diffusive flow. Unsteady flow through a moving vertical slender cylinder with the viscous dissipation offered by Patil et al. [14]. Murthy et al. [15] presented a parallel finite element study of 3D flow in a cubic porous enclosure. The unsteady flow over a vertical cone introduced by Roy et al. [16]. Ravindran and
Ganapathirao [17] presented non-uniform slot suction/injection over a vertical cone. Ganapathirao et al. [18] introduced a study of unsteady flow over a vertical cone with non-uniform slot suction (injection).

The impact of chemical reactions on the flow is also an imperative field because of its key role in the various sections like the design of equipment of hydrometallurgical and chemical processing industries, food processing plants, cooling towers, damage of crops for freezing, combustion processes and other engineering processes. Several researchers analyzed it on different bodies. We can see a few of which here. An experimental study of miscible viscous fingering with viscosity changes induced by variations was examined by Nagatsu et al. [19]. Patil and Kulkarni [20] introduced free convection flow through porous media of a polar fluid with internal heat generation. Flow over a vertical truncated cone with heat generation/absorption and variable viscosity effects on the double-diffusive convection analyzed by Mahdy [21]. Ravindran et al. [22] proposed unsteady MHD flow over a vertical cone with heat generation/absorption and non-uniform slot mass transfer. Unsteady flow over a wedge with suction/injection and heat generation/absorption introduced by Ganapathirao et al. [23]. We can view more relevant work on the chemical reaction effect [24-31].

The study of the combined impact of injection/suction and chemical reaction effects on the mixed convection flow across a vertical cone saturated porous medium in the presentation of double dispersion effect did not recognize so far and through this, we aim it. We code the set of equations of the flow model in MATLAB via the bivariate Chebyshev spectral collocation quasi-linearization method and the computations stated in the results and discussions section with the significant applications. It establishes the precision verification of the chosen numerical technique through a comparison with previously published Kumari et al. [32] computations and found to be in great assent. The residual analysis section also 
illustrated which unblocks convergence of the present results.

This work is organized in the following pattern: Section 2 presents the mathematical formulation of the model, section 3 proposes the numerical simulation of the problem, while section 4 unfolds the numerical computations and we concluded in section 5 .

\section{MATHEMATICAL FORMULATION}

Consider a vertical cone with half-angle $\delta$ saturated with Darcy-Brinkman porous model in a steady, laminar, mixed convection flow of an incompressible viscous fluid. The cone surface with its generator is aligned with $x$-axis and $y$-axis is normal to the surface; the flow moves in an upward direction, parallel to the axis of the cone. The walls of the cone are supposed to be permeable so that the fluid can inject/sucted with velocity $v_{w}(x)$. A schematic representation of the flow model and coordinates system is sketched in Figure 1.

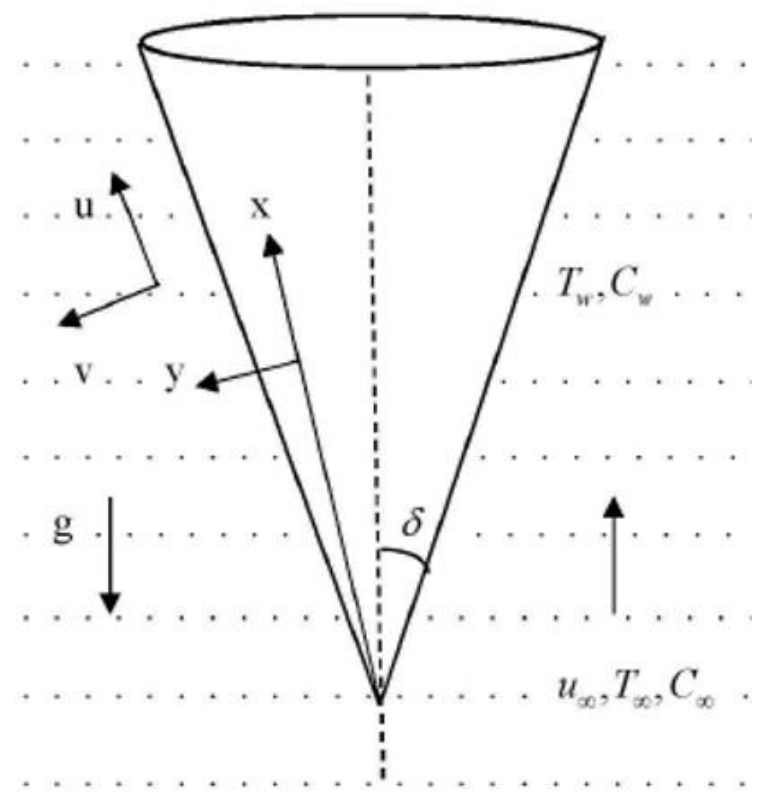

Figure 1. Flow model and coordinates system

The governing equations of the model are formulated as follows, by considering the work $[17,22,32]$.

$$
\begin{gathered}
\frac{\partial u}{\partial x}+\frac{\partial v}{\partial y}=0 \\
u \frac{\partial u}{\partial x}+v \frac{\partial u}{\partial y}=U \frac{d U}{d x}+\frac{\mu_{e}}{\rho} \frac{\partial^{2} u}{\partial y^{2}} \\
-\frac{\mu}{\rho K} u+g \cos \delta\left(\beta_{T}\left(\mathrm{~T}-\mathrm{T}_{\infty}\right)-\beta_{C}\left(C-C_{\infty}\right)\right) \\
u \frac{\partial T}{\partial x}+v \frac{\partial T}{\partial y}=\frac{\partial}{\partial y}\left(\alpha_{e} \frac{\partial T}{\partial y}\right) \\
u \frac{\partial C}{\partial x}+v \frac{\partial C}{\partial y}=\frac{\partial}{\partial y}\left(D_{e} \frac{\partial C}{\partial y}\right)-K_{c}\left(C-C_{\infty}\right)
\end{gathered}
$$

The boundary conditions are:

$$
\begin{gathered}
u=0, v=v_{w}, T(\mathrm{x}, \mathrm{y})=T_{w}, C(\mathrm{x}, \mathrm{y})=C_{w} \text { at } y=0 \\
u \rightarrow U=u_{\infty} x^{m}, T(\mathrm{x}, \mathrm{y}) \rightarrow T_{\infty}, C(\mathrm{x}, \mathrm{y}) \rightarrow C_{\infty}, \text { as } \\
\mathrm{y} \rightarrow \infty
\end{gathered}
$$

where, $u$ and $v$ are velocity components in $x$ and $y$ directions. $\delta$ is the half-angle of the cone, $T$ is the fluid temperature, $C$ is the concentration, $v$ is the kinematic viscosity, $g$ is the gravitational acceleration, $K$ is the permeability coefficient of the porous medium; $K_{c}$ is the chemical reaction rate, $\beta_{T}$ and $\beta_{C}$ are volumetric coefficients of the thermal and concentration expansions, respectively. $\alpha_{e}$ and $D_{e}$ are the effective thermal and Solutal diffusivities which can be write as $\alpha_{e}=\alpha+\gamma d u$ and $D_{e}=D+\zeta d u$, where $\alpha$ and $D$ are the molecular thermal and Solutal diffusivities, respectively.

Introducing the following non-dimensional variables:

$$
\begin{gathered}
\xi(\mathrm{x})=\left(\frac{2}{m+1} \frac{v x}{U}\right)^{1 / 2}, \\
\eta(\mathrm{x}, \mathrm{y})=y\left(\frac{m+1}{2} \frac{U}{v x}\right)^{1 / 2}, \\
\psi(\mathrm{x}, \mathrm{y})=\left(\frac{2}{m+1} v x U\right)^{1 / 2} f(\xi, \eta), \\
T(\mathrm{x}, \mathrm{y})=\left(T_{w}-T_{\infty}\right) \theta(\xi, \eta)+T_{\infty}, \\
C(\mathrm{x}, \mathrm{y})=\left(C_{w}-C_{\infty}\right) \phi(\xi, \eta)+C_{\infty}
\end{gathered}
$$

By the definition of stream function, we introduce as:

$$
u=\frac{\partial \psi}{\partial y} \text { and } v=-\frac{\partial \psi}{\partial x}
$$

Thus, we have:

$$
u=U f_{\eta}, \begin{aligned}
& v=-\frac{1}{2}\left(\frac{2}{m+1} \frac{v U}{x}\right)^{1 / 2} \\
& {\left[(\mathrm{~m}+1) \mathrm{f}+(\mathrm{m}-1)\left(\eta \mathrm{f}_{\eta}-\xi \mathrm{f}_{\xi}\right)\right]}
\end{aligned}
$$

Enforcing Eqns. (6), (7) and (8) into Eqns. (2)-(4), then got the following forms, respectively.

$$
\begin{gathered}
F^{\prime \prime}+\frac{2 m}{m+1}\left(1-F^{2}\right)+\mathrm{f} F^{\prime}-\frac{\xi^{2}}{D a} F \\
+\frac{2}{m+1} \frac{\lambda}{\operatorname{Pr}}(\theta+N \phi) \\
=\left(\frac{m-1}{m+1}\right) \xi\left(F^{\prime} \frac{\partial f}{\partial \xi}-F \frac{\partial F}{\partial \xi}\right) \\
\theta^{\prime \prime}+\operatorname{Prf} \theta^{\prime}+P e_{\gamma}\left(F \theta^{\prime \prime}+F^{\prime} \theta^{\prime}\right) \\
=\operatorname{Pr}\left(\frac{m-1}{m+1}\right) \xi\left(\theta^{\prime} \frac{\partial f}{\partial \xi}-F^{\frac{\partial \theta}{\partial \xi}}\right) \\
\phi^{\prime \prime}+S c \mathrm{f} \phi^{\prime}+P e_{\zeta}\left(F \phi^{\prime \prime}+F^{\prime} \phi^{\prime}\right)-\xi^{2} S c \Delta \phi \\
=S c\left(\frac{m-1}{m+1}\right) \xi\left(\phi^{\prime} \frac{\partial f}{\partial \xi}-F \frac{\partial \phi}{\partial \xi}\right)
\end{gathered}
$$

Boundary conditions Eq. (5) reduced as: 


$$
\begin{gathered}
F(\xi, \eta)=0, \quad \theta(\xi, \eta)=1, \phi(\xi, \eta)=1 \text { at } \eta=0 \\
F(\xi, \eta) \rightarrow 1, \theta(\xi, \eta) \rightarrow 0 \quad \phi(\xi, \eta) \rightarrow 0 \text { as } \eta \rightarrow \infty
\end{gathered}
$$

here primes represent derivatives with respect to $\eta$ and subscript $\xi$ represent partial derivatives, and $f=\int_{0}^{\eta} F d \eta+f_{w}$, $f_{w}$ is given as: $f_{w}+\left(\frac{1-m}{m+1}\right) \xi\left(f_{\xi}\right)_{w}=-\left(\frac{v_{w}}{v}\right) \xi=A \xi$;

where, $A=-\frac{v_{w}}{v}$ is the surface mass transfer parameter $\mathrm{A}<0$ and $\mathrm{A}>0$ are injection and suction, respectively; $\lambda=\frac{G r}{R e_{x}^{2}}$ is the buoyancy parameter, $N=\frac{\beta_{C}}{\beta_{T}}\left(\frac{C_{w}-C_{\infty}}{T_{w}-T_{\infty}}\right)$ is the buoyancy ratio, $R a_{x}=\frac{g K \beta_{T} x^{2}\left(T_{w}-T_{\infty}\right) \cos \gamma}{v \alpha}$ is the modified local Rayleigh number, $G r=\frac{R a_{x}}{P r}$ is the local Grashof number, $R e_{x}=\frac{U x}{v}$ is the local Reynold number, $\operatorname{Pr}=\frac{v}{\alpha_{m}}$ is the Prandtl number, $P e_{\gamma}=\frac{\gamma d U}{\alpha}$ is the thermal dispersion parameter, $P e_{\zeta}=\frac{\zeta d U}{D}$ is the thermal dispersion parameter, $S c=\frac{v}{D_{m}}$ is the Schmidt number, $\Delta=\frac{K_{C}}{v}$ is the chemical reaction parameter.

The principal bodily quantities of interest which can no longer be skipped are skin friction, heat and mass transfer rates in terms of local skin friction coefficient $C_{f}$, local Nusselt number $N u_{x}$, and local Sherwood number $S h_{x}$ can be written as: $C_{f}=\frac{2 \tau_{w}}{\rho U^{2}}, \quad N u_{x}=\frac{q_{w}}{\left(T_{w}-T_{\infty}\right)} \frac{x}{k}$ and $S h_{x}=\frac{q_{m}}{\left(C_{w}-C_{\infty}\right)} \frac{x}{D_{f}}$, here, $\tau_{w}=\mu\left(\frac{\partial u}{\partial y}\right)_{y=0}, q_{w}=-k_{e}\left(\frac{\partial T}{\partial y}\right)_{y=0}$ and $q_{m}=-D_{e}\left(\frac{\partial C}{\partial y}\right)_{y=0}$ are defined as wall skin friction, heat flux and mass flux, respectively.

Thus, we have

$$
\begin{gathered}
C_{f} \operatorname{Re}_{x}^{1 / 2}=2\left(\frac{m+1}{2}\right)^{1 / 2} F^{\prime}(\xi, 0) \\
N u_{x} \operatorname{Re}_{x}^{-1 / 2}=-\left(\frac{m+1}{2}\right)^{1 / 2} \\
\left(1+\operatorname{Pe}_{\gamma} F(\xi, 0)\right) \theta^{\prime}(\xi, 0) \\
S h_{x} \operatorname{Re}_{x}^{-1 / 2}=-\left(\frac{m+1}{2}\right)^{1 / 2} \\
\left(1+\operatorname{Pe}_{\zeta} F(\xi, 0)\right) \phi^{\prime}(\xi, 0)
\end{gathered}
$$

\section{NUMERICAL SOLUTION}

The non-similar equations are solved numerically through the well explained numerical technique call the bivariate Chebyshev spectral collocation quasi-linearization method by Motsa et al. [33], Meena et al. [34, 35]. To test the efficiency of the numerical technique; the residual analysis study is computed, which converges to $10^{-12}$.

\section{RESULTS AND DISCUSSIONS}

In this section, the outcomes for non-similarity solution of Eqns. (9-11) with boundary conditions Eq. (12) are displayed graphically and numerically via the above explained technique. The governing parameters are considered in the following ranges $0.66 \leq S c \leq 2.57,-1.0 \leq N \leq 1.0,0 \leq m \leq 1 / 2,0.733 \leq P r \leq 6.7$, $1.0 \leq \lambda \leq 7.0,0.5 \leq P e_{\gamma} \leq 2.0,-1.0 \leq \Delta \leq 1.0,0.5 \leq P e_{\zeta} \leq 2.0,-1.0 \leq A \leq 1.0$ and $0.0 \leq \xi \leq 1.0$. We carry the boundary layer region between 4.0 to 7.0 .

Figure 2 shows the residual analysis over iterations of $F(\xi$, $\eta), \theta(\xi, \eta)$ and $\phi(\xi, \eta)$ profiles for $D a$ variation with $m=1 / 2, P r=$ $0.733, \lambda=1.0, P e_{\gamma}=0.5, P e_{\zeta}=0.5, N=1.0$ and $\Delta=1.0$, respectively It establishes the convergence of present adopted numerical technique. In Figure 2 (a), we observe that the method converges after eleven iterations for the various values of $D a$ to $10^{-11}$, while in Figure 2 (b) the method converges after ten iterations for the various values of $D a$ to $10^{-11}$ and in Figure 2 (c) the method converges after twelve iterations for the various values of $D a$ to $10^{-12}$. This in turn validates the stability and convergence of the methods, and hence the results are accurate to approximately eleven digits.

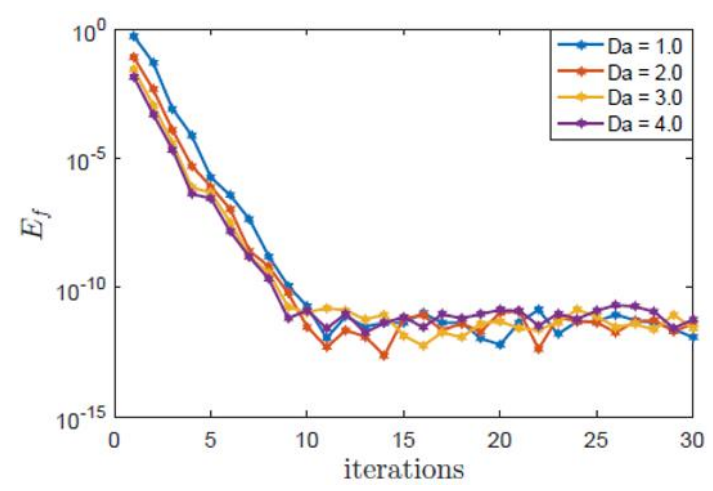

(a)

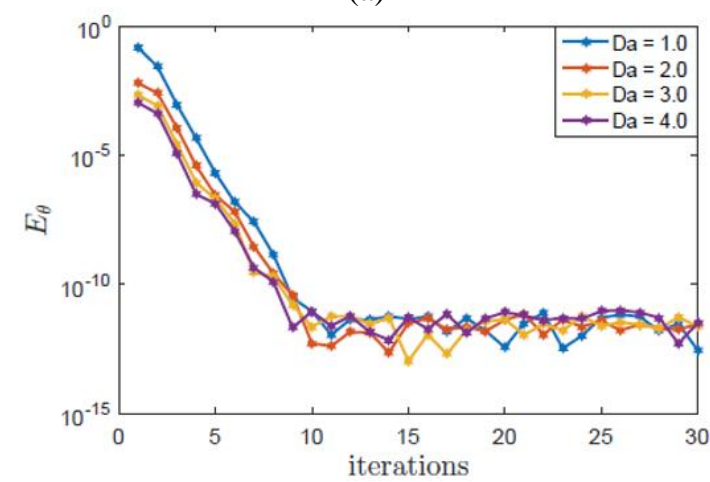

(b)

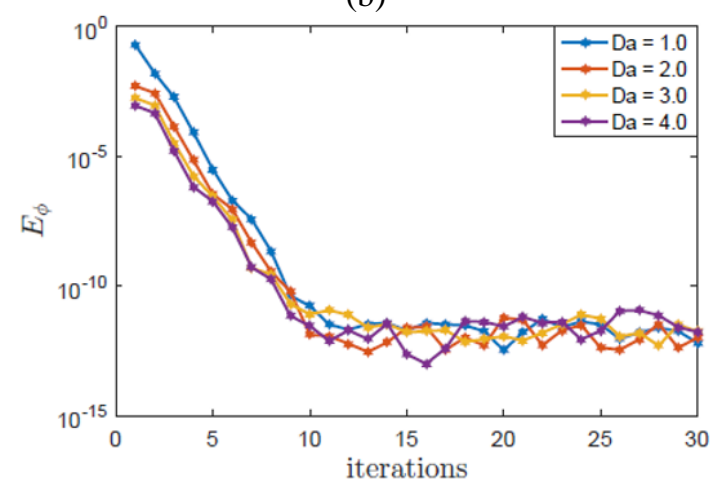

(c)

Figure 2. Residual analysis over iterations with $m=1 / 2$, $P r=0.733, \lambda=1.0, P e_{\gamma}=0.5, P e_{\zeta}=0.5, A=1.0, N=1.0$ and $\Delta=1.0$ are represented by (a), (b) and (c), respectively

Figure 3(a) exhibits a comparison of prior published computation with Kumari et al. [32] which authenticated validation of the chosen numerical technique. Figure 3(b, c) introduces the impact of $\operatorname{Pr}$ and $P e_{\gamma}$ together on the $F(\xi, \eta)$ 
and $\theta(\xi, \eta)$ profiles for $m=1 / 2, S c=2.57, \lambda=1.0, A=1.0, P e_{\zeta}=0.5$, $N=1.0$ and $\Delta=0.5$. Both the $F(\xi, \eta)$ and $\theta(\xi, \eta)$ profiles intensify with $P e_{\gamma}$ and clashing with $\mathrm{Pr}$. The intensification rate is greater with a small Prandtl number because of lower viscosity fluid. Therefore, thermal dispersion impact is more prevalent on low viscosity fluid. We perceive it from the velocity profile that the enhancement is less near to the surface of the cone because of the no-slip condition and increases far away and the similar trend concerned in the temperature profile.

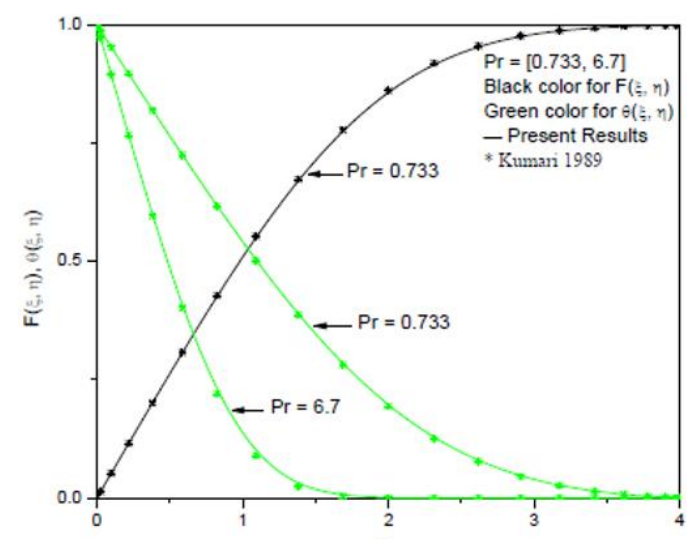

(a)

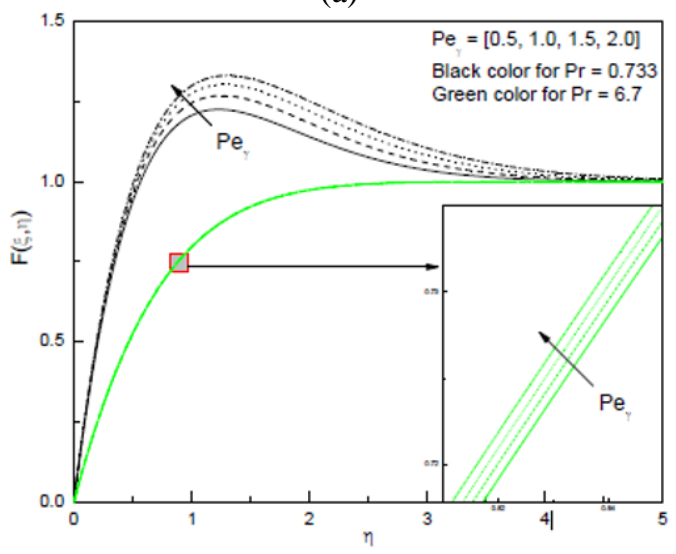

(b)

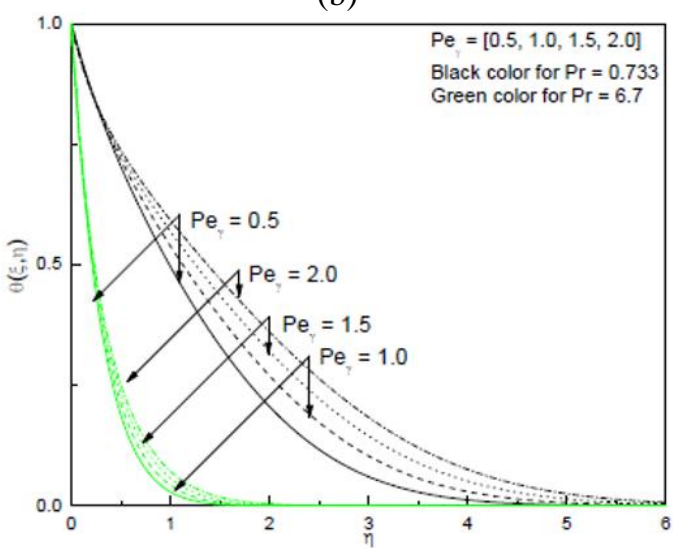

(c)

Figure 3. Comparison of the present results with Kumari 1989 of the $F(\xi, \eta)$ and $\theta(\xi, \eta)$ profiles for $m=0, S c=0, \lambda=0$,

$P e_{\gamma}=0, A=0, P e_{\zeta}=0, N=0$ and $\Delta=0$ represented by (a). Impression of $P r$ and $P e_{\gamma}$ together on the $F(\xi, \eta)$ and $\theta(\xi, \eta)$ with $m=1 / 2, S c=2.57, \lambda=1.0, D a=1.0, A=1.0, P e_{\zeta}=0.5, N=1.0$

and $\Delta=0.5$ are represented by (b) and (c), respectively

Figure 4 portrays the effect of $S c$ and $\Delta$ together on the $F(\xi$, $\eta), \theta(\xi, \eta)$ and $\phi(\xi, \eta)$ profiles with $m=1 / 2, \operatorname{Pr}=0.733, P e_{\gamma}=0.5$, $\lambda=1.0, A=1.0, P e_{\zeta}=0.5, N=1.0$ and $\Delta=0.5$. All the $F(\xi, \eta), \theta(\xi$, $\eta)$ and $\phi(\xi, \eta)$ profiles diminishes with $\Delta$ and it has more variation with small Schmidt number $S c=0.22$ which refers to the less viscosity of the fluid. The $\theta(\xi, \eta)$ profile does not have enormous variation with $\Delta$, therefore, the chemical reaction does not affect much on the temperature distribution of the flow.

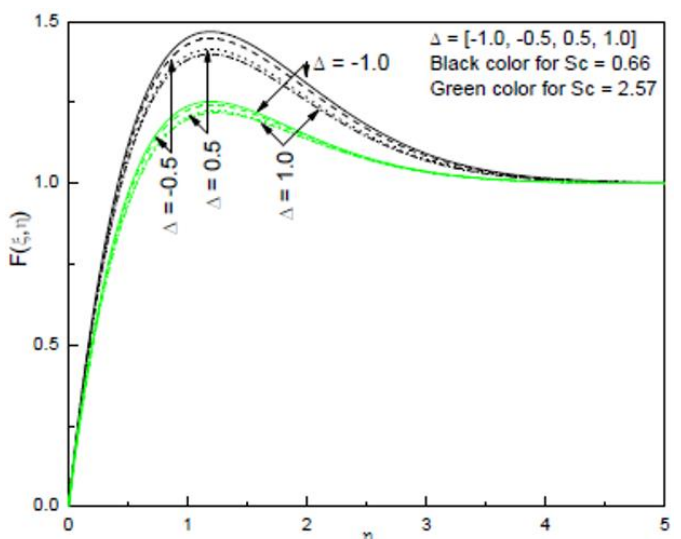

(a)

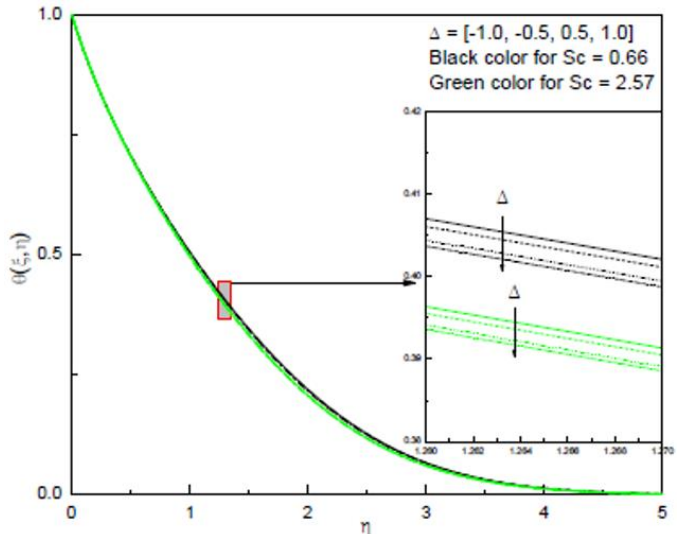

(b)

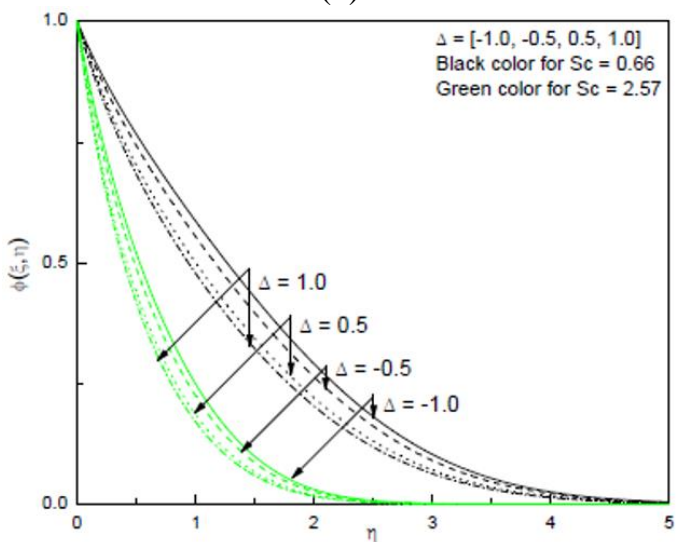

(c)

Figure 4. Impression of $S c$ and $\Delta$ together on $F(\xi, \eta), \theta(\xi, \eta)$ and $\phi(\xi, \eta)$ with $m=1 / 2, \operatorname{Pr}=0.733, \lambda=1.0, D a=1.0, A=1.0$, $P e_{\gamma}=0.5, P e_{\zeta}=0.5, N=1.0$ and $\Delta=0.5$ are represented by (a), (b) and (c), respectively

Figure 5 shows $F(\xi, \eta), \theta(\xi, \eta)$ and $\phi(\xi, \eta)$ profiles profiles for variation of the $S c$ and $A$ together with $m=1 / 2, \operatorname{Pr}=0.733$, $P e_{\gamma}=0.5, \lambda=1.0, P e_{\zeta}=0.5, N=1.0$ and $\Delta=0.5$. All the $F(\xi, \eta), \theta(\xi$, $\eta)$ and $\phi(\xi, \eta)$ profiles enhance with $A<0$ (injection) but converse with $A>0$ (suction), therefore, decreases. The physical cause of this can count as in the injection case the fluid injected from the cone which enhances the velocity of 
flow and the heat and concentration profiles also enhance but with the suction surface of the cone suck the fluid that reduces the velocity and the temperature and concentration profiles also reduces. It is also noted that if the viscosity of fluid increases then the impact of injection suction reduces, and it is greater with small $S c=0.66$.

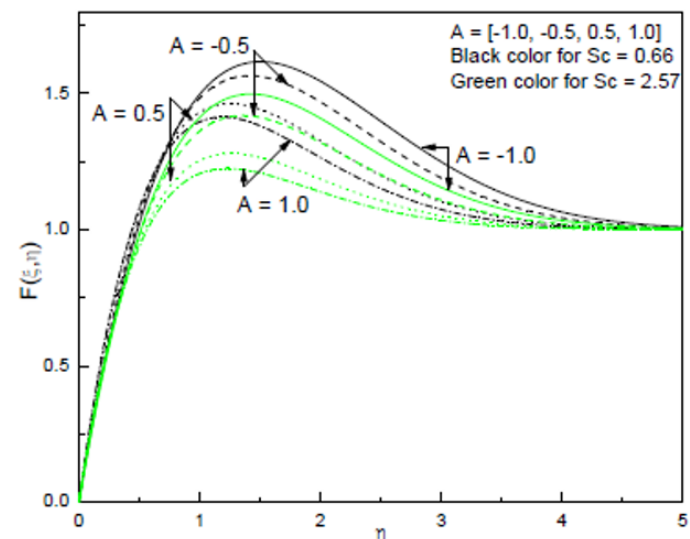

(a)

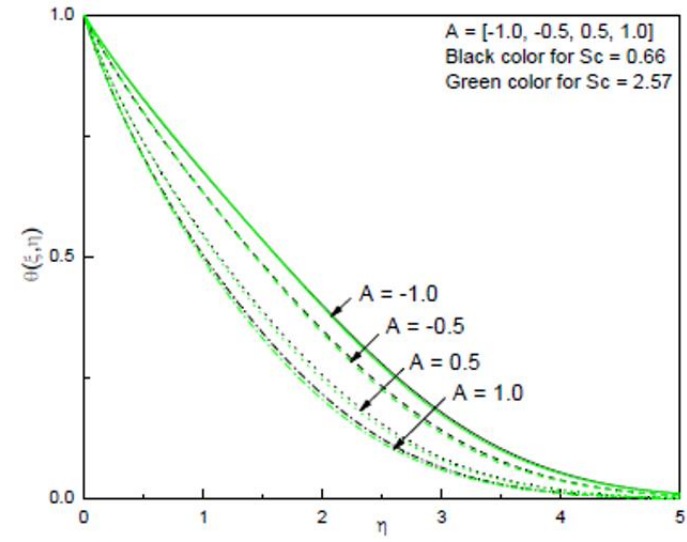

(b)

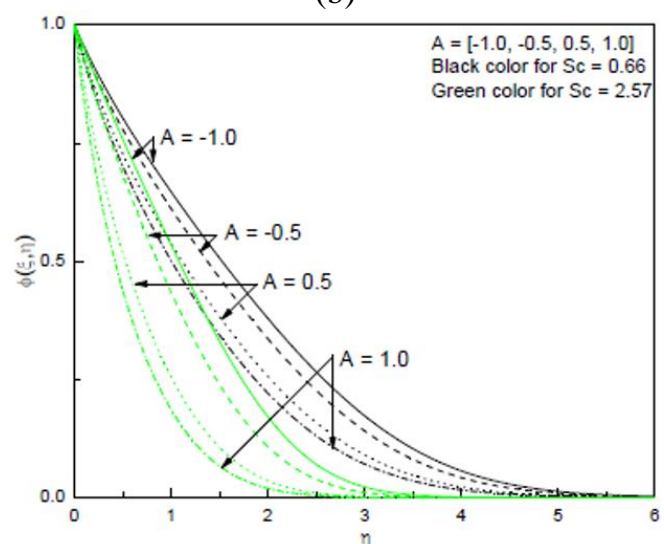

(c)

Figure 5. Impression of $S c$ and $A$ together on $F(\xi, \eta), \theta(\xi, \eta)$ and $\phi(\xi, \eta)$ with $m=1 / 2, \operatorname{Pr}=0.733, \lambda=1.0, D a=1.0, A=1.0$, $P e_{\gamma}=0.5, P e_{\zeta}=0.5, N=1.0$ and $\Delta=0.5$ are represented by (a), (b) and (c), respectively

Figure $6(\mathrm{a}, \mathrm{b})$ offers the impression of $P e_{\zeta}$ and $S c$ jointly on the $F(\xi, \eta)$ and $\phi(\xi, \eta)$ profiles regarding $m=1 / 2, \operatorname{Pr}=0.733$, $P e_{\gamma}=0.5, \lambda=1.0, A=1.0, N=1.0$ and $\Delta=0.5$. Both the $F(\xi, \eta)$ and $\phi(\xi, \eta)$ profiles enhances with $P e_{\zeta}$, away the surface of the cone, and not much dominant on the $F(\xi, \eta)$ profile near the surface because of the non-slip condition and similar followed by $\theta(\xi$, $\eta$ ) profile. Figure 6 (c) reflects the impact of axial distance $\xi$ on the $F(\xi, \eta), \theta(\xi, \eta)$ and $\phi(\xi, \eta)$ profiles and observed decrement in all the profiles with $\xi$.

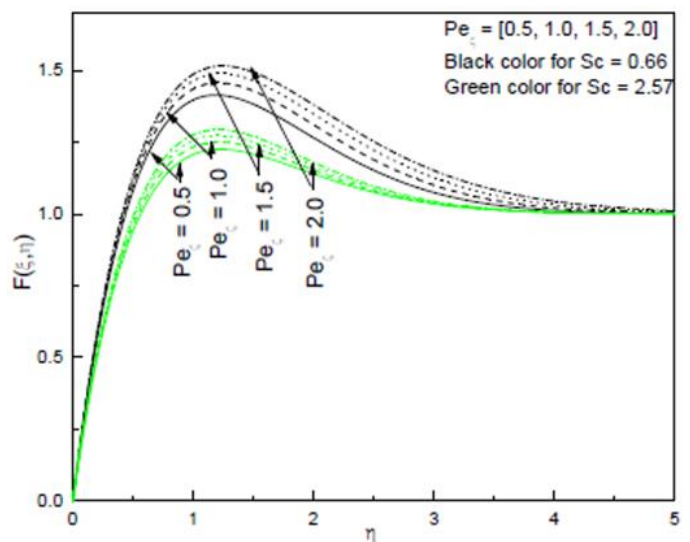

(a)

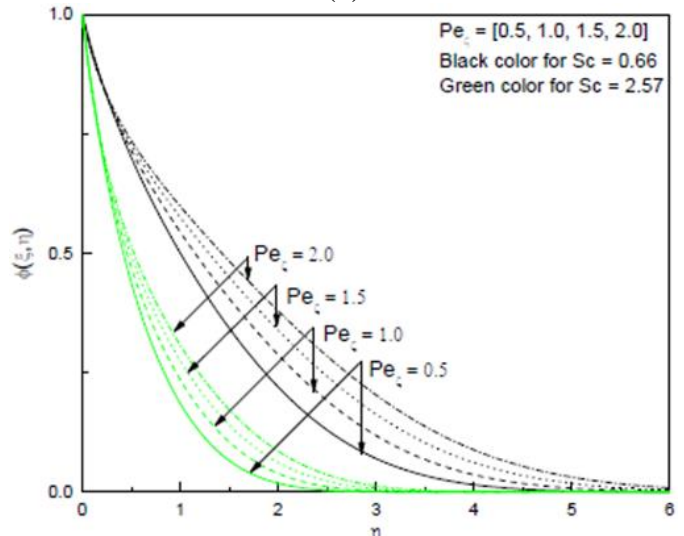

(b)

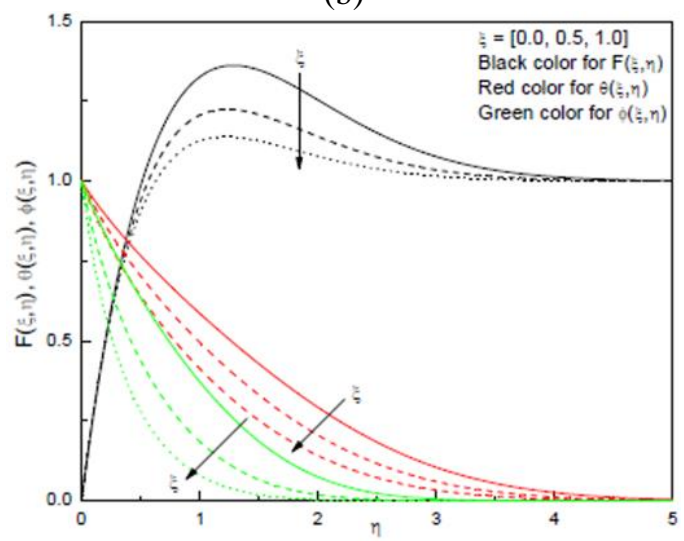

(c)

Figure 6. Impression of $S c$ and $P e_{\zeta}$ together on $F(\xi, \eta)$ and $\phi(\xi, \eta)$ with $m=1 / 2, \operatorname{Pr}=0.733, \lambda=1.0, D a=1.0, A=1.0$, $P e_{\gamma}=0.5, A=1.0, N=1.0$ and $\Delta=0.5$ are represented by (a) and (b), respectively. Impression of $\xi$ on $F(\xi, \eta), \theta(\xi, \eta)$ and $\phi(\xi$, $\eta)$ represented by (c)

Figure 7 introduces the $C_{f} R e_{x}^{1 / 2}, N u_{x} R e_{x}^{-1 / 2}$ and $S h_{x} R e_{x}^{-1 / 2}$ for mutation in $S c$ and $A$ together over the axial distance $\xi$ with $m=1 / 2, \operatorname{Pr}=0.733, D a=1.0, \Delta=0.5, P e_{\gamma}=0.5$, $P e_{\zeta}=0.5, N=1.0$ and $\lambda=1.0$. All properties fall with injection over axial distance, but contrary to suction therefore enhances. The impact of $A$ is more dominant on the $C_{f} R e_{x}^{1 / 2} \quad$ with low viscosity fluid, hence with low Schmidt number $(S c=0.66)$ but the $S h_{x} R e_{x}^{-1 / 2}$ is inimical, hence more dominant with the high Schmidt number $(S c=2.57)$ 


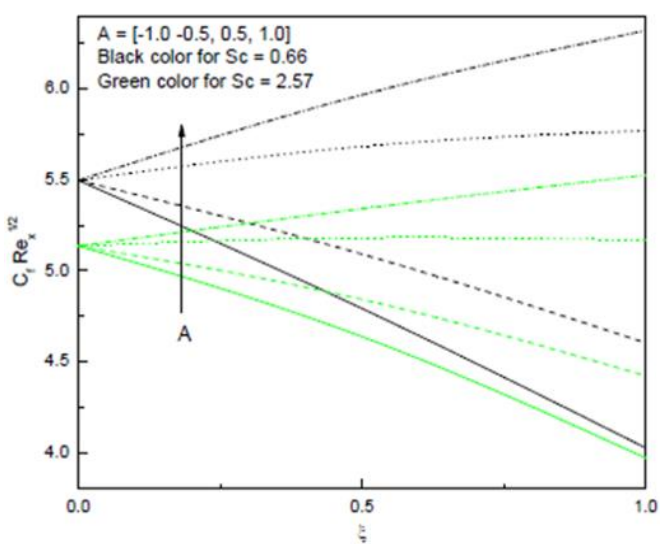

(a)

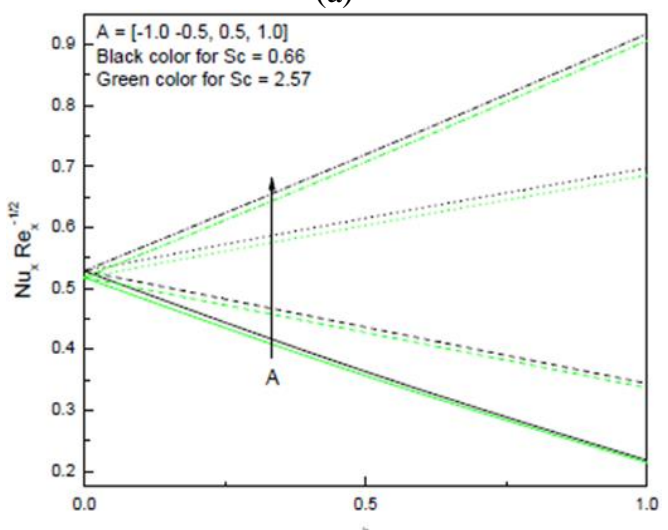

(b)

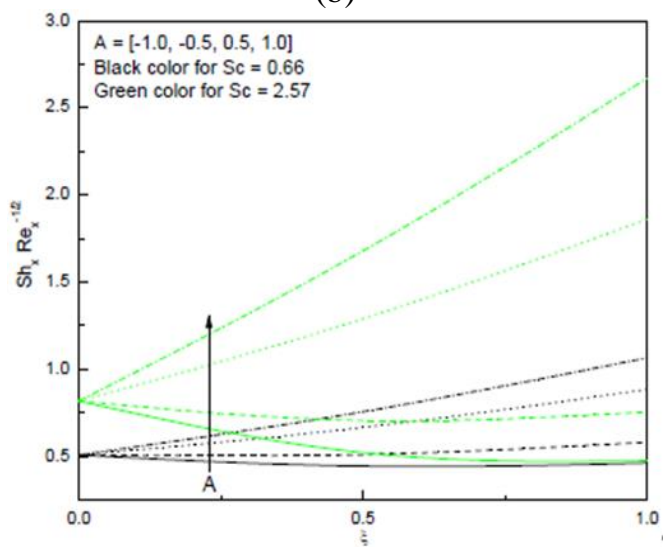

(c)

Figure 7. Impression of $S c$ and $A$ together on $C_{f} R e_{x}^{1 / 2}$, $N u_{x} R e_{x}^{-1 / 2}$ and $S h_{x} R e_{x}^{-1 / 2} \quad$ with $m=1 / 2, P r=0.733$, $\lambda=1.0, D a=1.0, P e_{\zeta}=0.5, P e_{\gamma}=0.5, N=1.0$ and $\Delta=0.5$ are represented by (a), (b) and (c), respectively

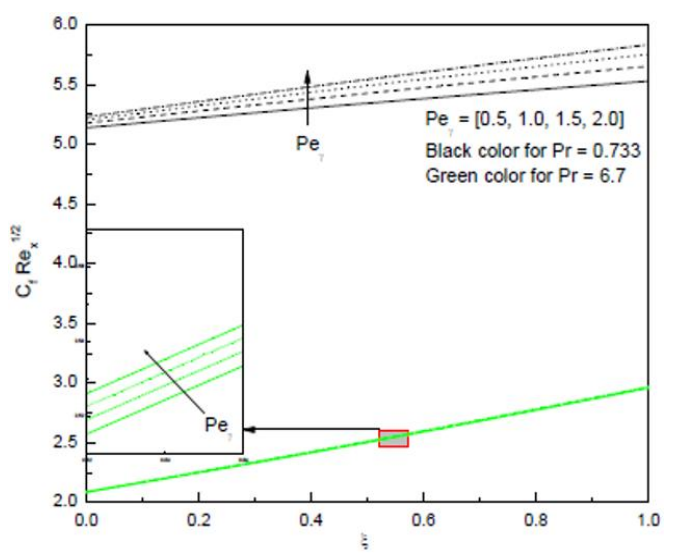

(a)

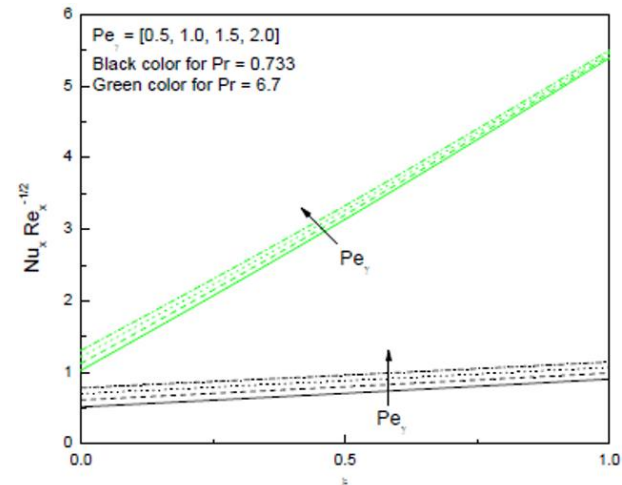

(b)

Figure 8. Impression of Pr and $P e_{\gamma}$ together on $C_{f} R e_{x}^{1 / 2}$ and $N u_{x} R e_{x}^{-1 / 2} \quad$ with $m=1 / 2, S c=2.57, \lambda=1.0, D a=1.0$, $P e_{\zeta}=0.5, A=1.0, N=1.0$ and $\Delta=0.5$ are represented by (a) and (b), respectively

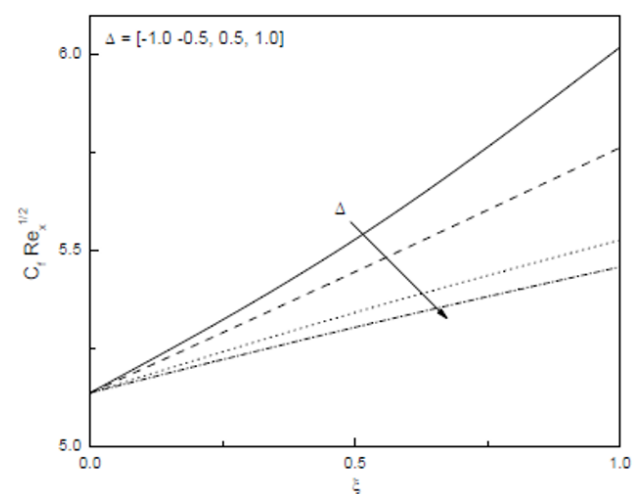

(a)

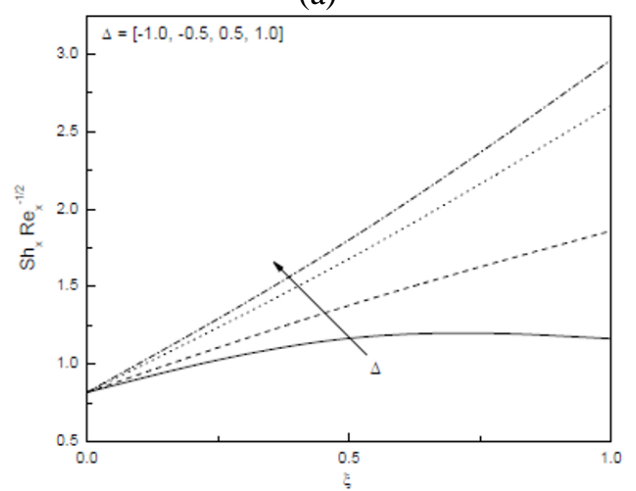

(b)

Figure 9. Impression of $\Delta$ on $C_{f} R e_{x}^{1 / 2}$ and $S h_{x} R e_{x}^{-1 / 2}$ with $m=1 / 2, S c=2.57, \lambda=1.0, D a=1.0, P e_{\gamma}=0.5, P e_{\zeta}=0.5$, $A=1.0$ and $N=1.0$ are represented by (a) and (b), respectively

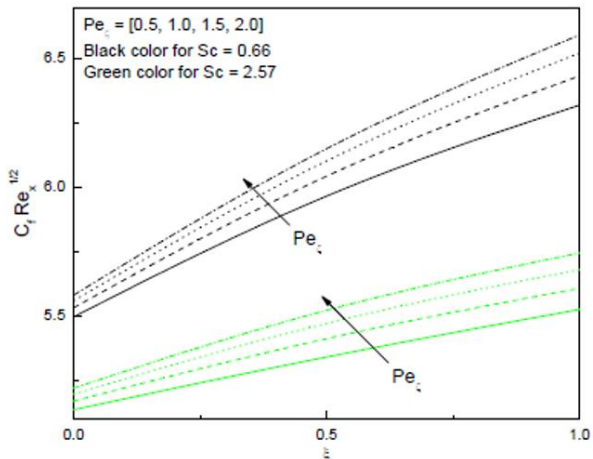

(a) 


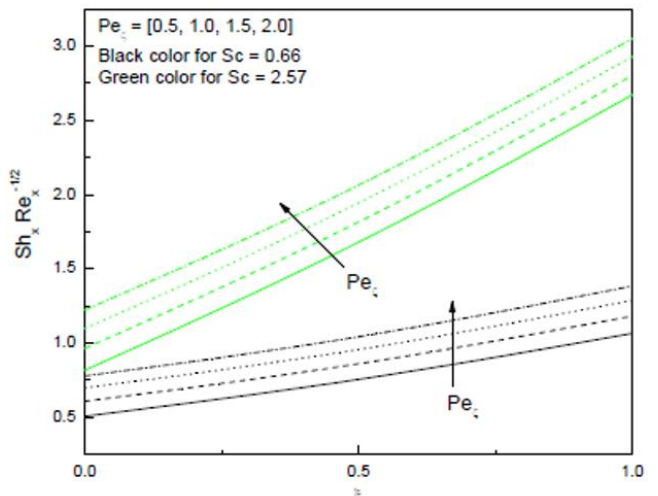

(b)

Figure 10. Impression of $S c$ and $P e_{\zeta}$ together on $C_{f} R e_{x}^{1 / 2}$ and $S h_{x} R e_{x}^{-1 / 2} \quad$ with $m=1 / 2, P r=0.733, \lambda=1.0, D a=1.0$, $P e_{\gamma}=0.5, \Delta=0.5, A=1.0$ and $N=1.0$ are represented by (a) and (b), respectively

Figure 8 presents the $C_{f} R e_{x}^{1 / 2}$ and $N u_{x} R e_{x}^{-1 / 2}$ for variation of $\mathrm{Pr}$ and $P e_{\gamma}$ together over axial distance $\xi$ with $m=1 / 2, S c=2.57, D a=1.0, \Delta=0.5, A=1.0, P e_{\zeta}=0.5, N=1.0$ and $\lambda=1.0$. Both the $C_{f} R e_{x}^{1 / 2}$ and $N u_{x} R e_{x}^{-1 / 2} \quad$ enhances with $P e_{\gamma}$ and hold similar tendency with the axial distance $\xi$. Figures 9 and 10 reflects $C_{f} R e_{x}^{1 / 2}$ and $S h_{x} R e_{x}^{-1 / 2}$ for variation in $\Delta$ and $P e_{\zeta}$ with $m=1 / 2, P r=0.733, D a=1.0, \Delta=0.5, A=1.0$, $P e_{\gamma}=0.5, N=1.0$ and $\lambda=1.0$, respectively. The $C_{f} R e_{x}^{1 / 2}$ diminishes with $\Delta$ over $\xi$ but $S h_{x} R e_{x}^{-1 / 2}$ is contradictory. Both the $C_{f} R e_{x}^{1 / 2}$ and $S h_{x} R e_{x}^{-1 / 2}$ enhances with $P e_{\zeta}$ over $\xi$.

Table 1 produces numerical computations of the skin friction coefficient $C_{f}$, local Nusselt number $N u_{x}$ and Sherwood number $S h_{x}$ for mutation of the Prandtl number Pr, Schmidt number $S c$, buoyancy parameters $\lambda$, buoyancy ratio $N$, chemical reaction parameter $\Delta$, surface mass transfer $A$, thermal dispersion parameter $P e_{\gamma}$ and Solutal dispersion parameter $P e_{\zeta}$, respectively. An increment in Pr decrease $C_{f}$ and $S h_{x}$ but $N u_{x}$ is contradictory. Likewise, $S c$ diminishes $C_{f}$ and $N u_{x}$ but $S h_{x}$ is inconsistent. $\lambda$ and $N$ both enhance all the $C_{f}, N u_{x}$ and $S h_{x}$ properties. $C_{f}$ and $N u_{x}$ diminishes with $\Delta$ but $S h_{x}$ is in contrary trend, i.e. enhances. All the $C_{f}, N u_{x}$ and $S h_{x}$ increases with $A, P e_{\gamma}$ and $P e_{\zeta}$, respectively.

Table 1. Influence of $\operatorname{Pr}, S c, \lambda, N, \Delta, A$ and $P e_{\gamma}$ on $C_{f}, N u_{x}$ and $S h_{x}$ with respect to $\operatorname{Pr}=0.733, \lambda=1.0, P e_{\gamma}=0.5, S c=2.57, \mathrm{~A}=1.0$, $\mathrm{N}=1.0$ and $\Delta=0.5$

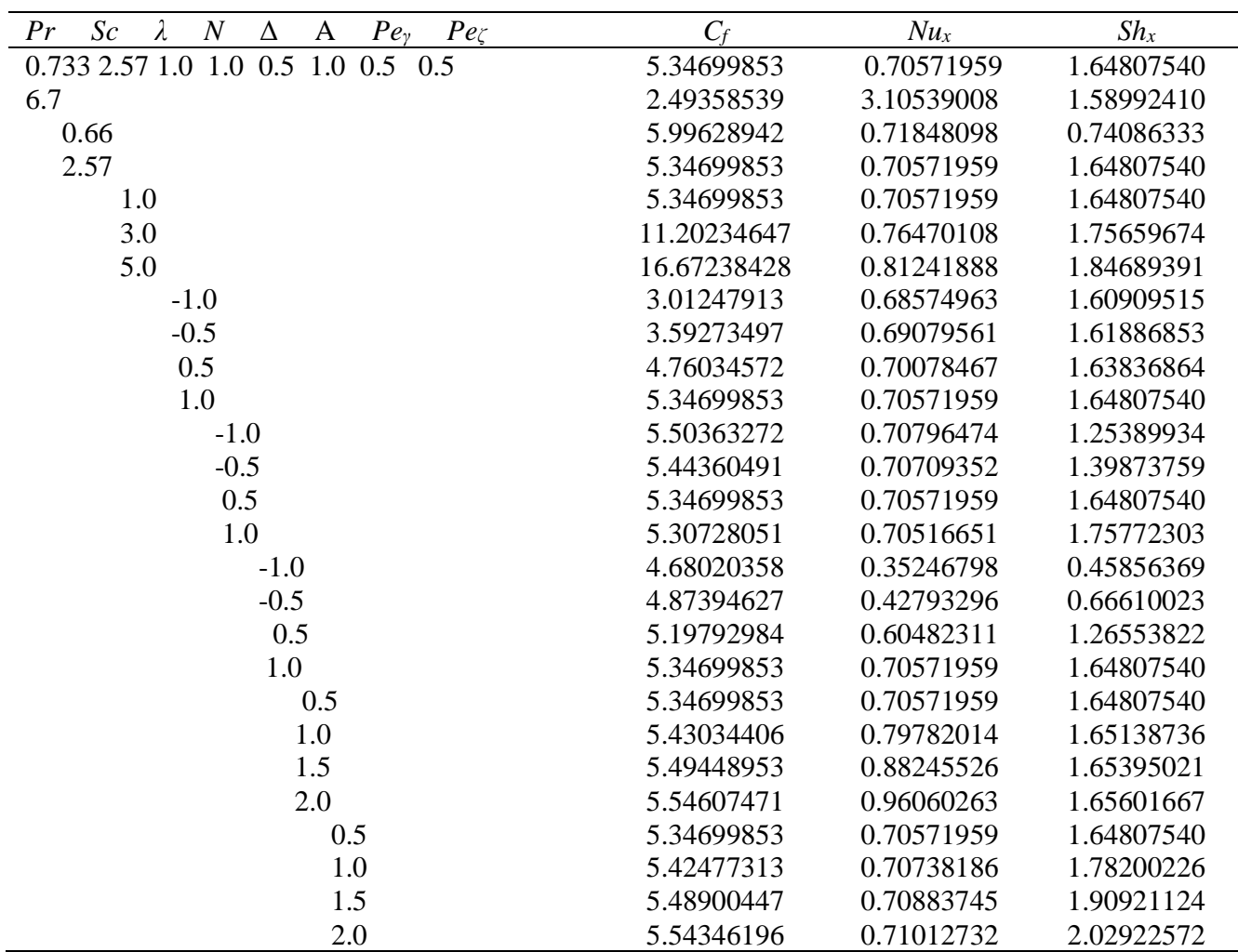

\section{CONCLUSION}

This manuscript aspires to investigate the influence of the injection/suction effects because of mixed convection flow in the presence of double dispersion and chemical reaction effects across a vertical cone saturated porous medium of a steady two-dimensional viscous incompressible fluid. The numerical computations figured and tabulated through the described technique, and we describe characteristics in the above section. The buoyancy parameter and buoyancy ratio both upgrade the $C_{f}, N u_{x}$ and $S h_{x}$, respectively. Likewise, all the $C_{f}, N u_{x}$ and $S h_{x}$ increases with $A, P e_{\gamma}$ and $P e_{\zeta}$, respectively. We perceive it from the velocity profile that the enhancement is less near to the surface of the cone because of the no-slip condition and increases far away and the similar trend concerned in the temperature and concentration profiles. $C_{f}$ and $N u_{x}$ diminishes with $\Delta$ but $S h_{x}$ enhances and noted that $\Delta$ is prevailing on the concentration profile and mass transfer and does not interrupt temperature distribution in the flow. An increment in $\operatorname{Pr}$ decrease $C_{f}$ and $S h_{x}$ but $N u_{x}$ increases. 
Likewise, $S c$ diminishes $C_{f}$ and $N u_{x}$ but $S h_{x}$ enhances. All injection/suction, double dispersion, and chemical reaction impacts are more dominant in less viscosity fluid $(\operatorname{Pr}=0.733$, $S c=0.66)$ comparing to high $(\operatorname{Pr}=6.7, S c=2.57)$.

\section{REFERENCES}

[1] Aldoss, T.K., Al-Nimr, M.A., Jarrah, M.A., Al-Sha'er, B.J. (1995). Magnetohydrodynamic mixed convection from a vertical plate embedded in a porous medium. Numerical Heat Transfer, Part A: Applications, 28(5): 635-645. https://doi.org/10.1080/10407789508913766

[2] Khanafer, K.M., Chamkha, A.J. (1999). Mixed convection flow in a lid-driven enclosure filled with a fluid-saturated porous medium. International Journal of Heat and Mass Transfer, 42(13): 2465-2481. https://doi.org/10.1016/S0017-9310(98)00227-0

[3] Kumari, M., Takhar, H.S., Nath, G. (2001). Mixed convection flow over a vertical wedge embedded in a highly porous medium. Heat and Mass Transfer, 37(2): 139-146. https://doi.org/10.1007/s002310000154

[4] Aydın, O., Kaya, A. (2008). Radiation effect on MHD mixed convection flow about a permeable vertical plate. Heat and Mass Transfer, 45(2): 239-246. https://doi.org/10.1007/s00231-008-0428-y

[5] Cimpean, D., Pop, I., Ingham, D.B., Merkin, J.H. (2009). Fully developed mixed convection flow between inclined parallel plates filled with a porous medium. Transport in Porous Media, 77(1): 87-102. https://doi.org/10.1007/s11242-008-9264-2

[6] Mukhopadhyay, S. (2012). Mixed convection boundary layer flow along a stretching cylinder in porous medium. Journal of Petroleum Science and Engineering, 96: 7378. https://doi.org/10.1016/j.petrol.2012.08.006

[7] Harris, S.D., Ingham, D.B., Pop, I. (2009). Mixed convection boundary-layer flow near the stagnation point on a vertical surface in a porous medium: Brinkman model with slip. Transport in Porous Media, 77(2): 267 285. https://doi.org/10.1007/s11242-008-9309-6

[8] Meena, O.P. (2020). Mixed convection nanofluid flow over a vertical wedge saturated in porous media with the influence of thermal dispersion using Lie group scaling. Computational Thermal Sciences: An International Journal, 12(3): 91-105. https://doi.org/10.1615/ComputThermalScien.20200323 30

[9] Mukhopadhyay, S. (2009). Effect of thermal radiation on unsteady mixed convection flow and heat transfer over a porous stretching surface in porous medium. International Journal of Heat and Mass Transfer, 52(1314):

3261-3265 https://doi.org/10.1016/j.ijheatmasstransfer.2008.12.029

[10] Meena, O.P. (2020). Mixed convection nanofluid flow over a vertical wedge saturated in a porous medium with influence of double dispersion using lie group scaling. Special Topics \& Reviews in Porous Media: An International Journal, 11(3): 297-311. https://doi.org/10.1615/SpecialTopicsRevPorousMedia. 2020031755

[11] Cimpean, D.S., Pop, I. (2012). Fully developed mixed convection flow of a nanofluid through an inclined channel filled with a porous medium. International Journal of Heat and Mass Transfer, 55(4): 907-914. https://doi.org/10.1016/j.ijheatmasstransfer.2011.10.018

[12] Kumar, B.R., Murthy, S.K. (2010). Mixed convection in a non-Darcian fluid saturated square porous enclosure under multiple suction effect. International Journal of Heat and Mass Transfer, 53(25-26): 5764-5773. https://doi.org/10.1016/j.ijheatmasstransfer.2010.08.006

[13] Daba, M., Devaraj, P. (2017). Unsteady double diffusive mixed convection flow over a vertically stretching sheet in the presence of suction/injection. Journal of Applied Mechanics and Technical Physics, 58(2): 232-243. https://doi.org/10.1134/S0021894417020067

[14] Patil, P.M., Roy, S., Chamkha, A.J., Kulkarni, P.S. (2011). Unsteady mixed convection flow from a moving vertical slender cylinder in the presence of viscous dissipation. International Journal of Microscale and Nanoscale Thermal and Fluid Transport Phenomena, 2(4): 281.

[15] Murthy, S.K., Kumar, B.R., Nigam, M. (2015). A parallel finite element study of 3D mixed convection in a fluid saturated cubic porous enclosure under injection/suction effect. Applied Mathematics and Computation, 269: 841-862. https://doi.org/10.1016/j.amc.2015.07.028

[16] Roy, S., Datta, P., Mahanti, N.C. (2007). Non-similar solution of an unsteady mixed convection flow over a vertical cone with suction or injection. International Journal of Heat and Mass Transfer, 50(1-2): 181-187. https://doi.org/10.1016/j.ijheatmasstransfer.2006.06.024

[17] Ravindran, R., Ganapathirao, M. (2013). Non-uniform slot suction/injection into mixed convection boundary layer flow over vertical cone. Applied Mathematics and Mechanics, 34(11): 1327-1338. https://doi.org/10.1007/s10483-013-1748-7

[18] Ganapathirao, M., Revathi, G., Ravindran, R. (2014). Unsteady mixed convection boundary layer flow over a vertical cone with non-uniform slot suction (injection). Meccanica, $49(3)$ : 673-686. https://doi.org/10.1007/s11012-013-9819-3

[19] Nagatsu, Y., Matsuda, K., Kato, Y., Tada, Y. (2007). Experimental study on miscible viscous fingering involving viscosity changes induced by variations in chemical species concentrations due to chemical reactions. Journal of Fluid Mechanics, 571: 475-493. https://doi.org/10.1017/S0022112006003636

[20] Patil, P.M., Kulkarni, P.S. (2008). Effects of chemical reaction on free convective flow of a polar fluid through a porous medium in the presence of internal heat generation. International Journal of Thermal Sciences, 47(8): 1043-1054. https://doi.org/10.1016/j.ijthermalsci.2007.07.013

[21] Mahdy, A. (2010). Effect of chemical reaction and heat generation or absorption on double-diffusive convection from a vertical truncated cone in porous media with variable viscosity. International Communications in Heat and Mass Transfer, 37(5): 548-554. https://doi.org/10.1016/j.icheatmasstransfer.2010.01.00 7

[22] Ravindran, R., Ganapathirao, M., Pop, I. (2014). Effects of chemical reaction and heat generation/absorption on unsteady mixed convection MHD flow over a vertical cone with non-uniform slot mass transfer. International Journal of Heat and Mass Transfer, 73: 743-751. https://doi.org/10.1016/j.ijheatmasstransfer.2014.02.053

[23] Ganapathirao, M., Ravindran, R., Momoniat, E. (2015). 
Effects of chemical reaction, heat and mass transfer on an unsteady mixed convection boundary layer flow over a wedge with heat generation/absorption in the presence of suction or injection. Heat and Mass Transfer, 51(2): 289-300. https://doi.org/10.1007/s00231-014-1414-1

[24] Meena, O.P., Pranitha, J. (2020). Power-law nanofluid on mixed convection with influence of double dispersion saturated non-Darcy porous media. In AIP Conference Proceedings, 2246(1):

020019. https://doi.org/10.1063/5.0014503

[25] Mallikarjuna, B., Rashad, A.M., Chamkha, A.J., Raju, S.H. (2016). Chemical reaction effects on MHD convective heat and mass transfer flow past a rotating vertical cone embedded in a variable porosity regime. Afrika Matematika, 27(3): 645-665. https://doi.org/10.1007/s13370-015-0372-1

[26] Pranitha, J., Meena, O.P. (2020). Influence of double dispersion on natural convection flow over a vertical cone saturated porous media with Soret and Dufour effects. Int. J. Eng. Sci.(IJES), 2: 09-15.

[27] Srinivasacharya, D., Shafeeurrahman, M. (2017). Joule heating effect on entropy generation in MHD mixed convection flow of chemically reacting nanofluid between two concentric cylinders. International Journal of Heat and Technology, 35(1): 487-497. https://doi.org/10.18280/ijht.350305

[28] Meena, O.P., Janapatla, P., Chamkha, A.J. (2021). Influence of soret and dufour effects on mixed convection flow over a vertical cone with injection/suction effects. Journal of Porous Media, 24(4). https://doi.org/10.1615/JPorMedia.2021034700
[29] Sambath, P., Pullepu, B., Hussain, T., Shehzad, S.A. (2018). Radiated chemical reaction impacts on natural convective MHD mass transfer flow induced by a vertical cone. Results in Physics, 8: 304-315. https://doi.org/10.1016/j.rinp.2017.12.005

[30] Meena, O.P. (2021). Mixed convection flow over a vertical cone with double dispersion and chemical reaction effects. Heat Transfer, 50(5): 4516-4534. https://doi.org/10.1002/htj.22086

[31] Nayak, B., Mishra, S.R., Krishna, G.G. (2019). Chemical reaction effect of an axisymmetric flow over radially stretched sheet. Propulsion and Power Research, 8(1): 79-84. https://doi.org/10.1016/j.jppr.2019.01.002

[32] Kumari, M., Pop, I., Nath, G. (1989). Mixed convection along a vertical cone. International Communications in Heat and Mass Transfer, 16(2): 247-255. https://doi.org/10.1016/0735-1933(89)90026-2

[33] Motsa, S.S., Magagula, V.M., Sibanda, P. (2014). A bivariate Chebyshev spectral collocation quasilinearization method for nonlinear evolution parabolic equations. The Scientific World Journal, 2014. https://doi.org/10.1155/2014/581987

[34] Meena, O.P. (2019). Spectral quasi-linearization method for a porous channel problem with micropolar flow. A Journal of Composition Theory, 12: 40-45.

[35] Meena, O.P., Janapatla, P., Srinivasacharya, D. (2021). Mixed convection fluid flow over a vertical cone saturated porous media with double dispersion and injection/suction effects. International Journal of Applied and Computational Mathematics, 7(3): 1-20. https://doi.org/10.1007/s40819-021-00990-y 